\title{
Compared with the Effects of Two Measurement Methods on the Luminescent Properties of LED Phosphors
}

\author{
Zhaoyang Zhang ${ }^{1}$, Pingfan Ning ${ }^{1}$ \\ ('School of Electrial Engineering and Automation, Tianjin Polytechnic University, Tianjin 300387, China)
}

\begin{abstract}
Accurate measurement of LED phosphor performance parameters, the traditional test method commonly used is to use a laser or xenon lamp as an incident light source, which is not only expensive, but also the results are not accurate. While the use of LED chips as its incident light source, is not only cheap, but also closer to the real life of the application. And in practical applications, LED chip and phosphor are usually packaged and measured, but the packaging method is complex, the packaging technology is not advanced, which directly affect the optical performance of white LED.As a result, in this paper, another way with the blue LED chip directly stimulate the phosphor and measure its luminous performance, and comparative analysis of the two different ways, Concluded as follow: When using the blue LED directly stimulate the yellow phosphor test method, the measured phosphor external quantum efficiency and energy efficiency is higher, and the luminous performance of the phosphor is better, and the application of the industry has played a reference role.

Keywords: Phosphor; Blu-ray LED chip; External quantum efficiency; Energy efficiency
\end{abstract}

\section{Introduction}

As the core of solid-state lighting, LED (Light Emitting Diodes) with its energy saving, environmental protection, long life, strong structure of the unique advantages rapid access to the field of lighting, a new generation of "green lighting" ${ }^{[1]}$.In the preparation of white LED, the most mainstream way is to use LED chip to stimulate the yellow phosphor YAG: $\mathrm{Ce} 3+$,to produce white ${ }^{[2]}$.In the practical application, the two are usually used after packaging, but the packaging process is cumbersome, complex technology, resulting in the test phosphor luminous efficiency, external quantum efficiency and energy efficiency and some performance parameters, because the packaging technology is not perfect, So that the measured performance parameters of the phosphor is not accurate. Therefore, this paper uses the blue LED chip to directly stimulate the yellow phosphor YAG: $\mathrm{Ce} 3$ + way, the phosphor luminous efficiency, external quantum efficiency and energy efficiency and other performance parameters were measured, and analysis of the two different excitation methods. The measurement system uses the integration of the ball, spectrometer, fiber and computer hardware facilities, respectively, two different ways to stimulate the excitation ${ }^{[3]}$, And the measured luminous spectrum of the comparison, the use of the relevant formula to calculate the two ways of the luminous efficiency of the phosphor, the external quantum efficiency and energy efficiency, Finally, it is concluded that when the LED chip is used to directly excite the phosphor, the external quantum efficiency and energy efficiency of the phosphor are higher, the luminescent performance of the phosphor is stronger, which provides a reference for the better application of LED light source.

\section{Led Phosphor Performance Measurement Principal}

Many parameters can characterize the performance of phosphors used in white LEDs, such as spectral distribution, relative brightness, conductivity, etc. In general, the relative brightness is chosen as the evaluation standard for its luminous efficiency. The relative brightness is the ratio of the brightness of the sample phosphor to the brightness of the standard phosphor. It is only applicable to the evaluation of two color temperature and spectral distribution similar to the phosphor The performance. But the actual characterization of this method is not strong, the need for reference to the standard phosphor, and the standard phosphor is not easy to obtain, the preparation, storage conditions demanding. When the two kinds of phosphor color temperature, spectral power distribution is very different, the relative brightness to lose the actual measurement of significance. Therefore, in practical cases, with the luminous efficiency, external quantum efficiency and energy efficiency to assess the optical properties of phosphor ${ }^{[4-5]}$.

The luminous efficiency of phosphors $\eta \mathrm{L}$ : The ratio of the luminous flux value $\Phi \mathrm{V}(\mathrm{lm})$ generated after stimulating the "human eye" to the optical power of the light source $\Phi \mathrm{E}(\mathrm{mW})$ for exciting the phosphor is stimulated by the light radiation flux emitted by the phosphor stimulated by a certain band, The unit is $1 \mathrm{~m} / \mathrm{W}$. 


$$
\eta_{L}=\frac{\varphi_{V}}{\varphi_{E}}=\frac{683 \int_{\lambda_{1}}^{\lambda_{2}} \varphi_{e m}\left(\lambda_{e m}\right) V\left(\lambda_{e m}\right) d \lambda_{e m}}{\int_{\lambda_{1}}^{\lambda_{2}} \varphi_{e x}\left(\lambda_{e x}\right) d \lambda_{e x}}
$$

The external quantum efficiency of the phosphor $\eta \mathrm{Q}$ : Refers to the light stimulation in a certain band, the phosphor stimulated by the emitted light in the number of photons and the amount of photons contained in the ratio.

$$
\eta_{Q}=\frac{N_{\text {发射 }}}{N_{\text {激发 }}}=\frac{\int_{\lambda_{1}}^{\lambda_{2}} \varphi_{e m}\left(\lambda_{e m}\right) /\left(\frac{h c}{\lambda_{e m}}\right) d \lambda_{e m}}{\int_{\lambda_{1}}^{\lambda_{2}^{\prime}} \varphi_{e x}\left(\lambda_{e x}\right) /\left(\frac{h c}{\lambda_{e x}}\right) d \lambda_{e x}}
$$

Energy efficiency of phosphors $\eta \mathrm{E}$ : Refers to the phosphor in a certain band of light stimulation, the phosphor excited by the light after the light power $\Phi$ e and excited phosphor light optical power $\Phi e^{\prime}$ the ratio.

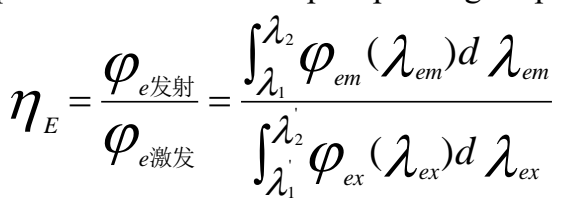

In this formula, $\lambda_{e x}$ is the wavelength of the excitation light, $\lambda_{e m}$ is the wavelength of the emitted light, $\varphi_{e m}\left(\lambda_{e m}\right)$ and $\varphi_{e x}\left(\lambda_{e x}\right)$ are the emission energy spectrum and the excitation energy spectrum of the phosphor, $\lambda_{1}-\lambda_{2}$ are the wavelength range of the emission spectrum, $\lambda_{1}^{\prime}-\lambda_{2}^{\prime}$ is the wavelength range of the excitation spectrum ${ }^{[6]}$.

\section{Experiment}

This experiment uses the integrating sphere, the astronomical photoelectric SSP8810-S light color thermoelectric comprehensive test system and the computer and so on equipment measurement system, as shown in Figure 1.The system is divided into three parts: integrating sphere system,SSP8810-S light color thermoelectric integrated test system, computer measurement system ${ }^{[7]}$.Excitation light source using $1 \mathrm{~W}$ high-power blue LED chip, phosphor using YAG: Ce3 + as a test object, in order to ensure its stability, the need to solder blue LED chip on the six-foot aluminum plate ${ }^{[8]}$. Figure 2 shows the LED lamp beads used in the direct illumination measurement before encapsulation. Figure 3 shows the lamp beads used to encapsulate the LED chip and the phosphor.

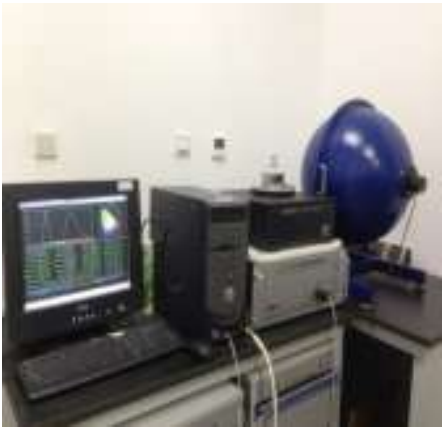

Figure 1 Measuring system

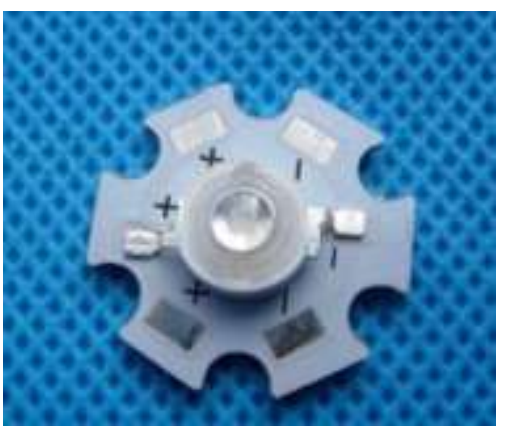

Figure 2 before packaging

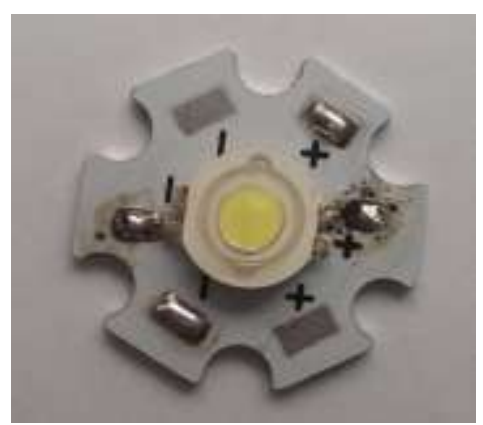

Figure 3 after packaging

In this experiment, the luminous performance of the phosphor was measured in four steps:

一. Test single blue LED chip, respectively, at $50 \mathrm{~mA}, 100 \mathrm{~mA}, 200 \mathrm{~mA}, 300 \mathrm{~mA}, 400 \mathrm{~mA}, 500 \mathrm{~mA}$ and other current under the spectral power distribution map;

二. The blue phosphor was directly excited by the blue LED chip to measure the white power emitted by the excited phosphor, and the spectral power distribution at 50mA, $100 \mathrm{~mA}, 200 \mathrm{~mA}, 300 \mathrm{~mA}, 400 \mathrm{~mA}, 500 \mathrm{~mA}$ and other current ${ }^{[9]}$

三. The blue power LED chip and phosphor package ${ }^{[10]}$, measured after the package issued by the white light, at $50 \mathrm{~mA}, 100 \mathrm{~mA}, 200 \mathrm{~mA}, 300 \mathrm{~mA}, 400 \mathrm{~mA}, 500 \mathrm{~mA}$ and other current under different spectral power 
distribution.

四. Compared with the above two methods of spectral power distribution map, the luminous efficiency of the phosphor, the external quantum efficiency and energy efficiency trends, analysis of which way the phosphor luminescent performance is more superior.

\subsection{Blue - Light Excitation Spectra at Different Driving Currents \\ III. Experimental Results And Analysis}

Figure 4 shows the excitation spectra of blue LEDs at 50mA, 100mA, 200mA, 300mA, 400mA, $500 \mathrm{~mA}$ drive current. According to the spectrum, it can be seen that the luminous intensity of the spectrum increases with the increase of the driving current. When the current is small, the increase of the luminous intensity is much larger than that in the case of large current. This is because with the test time, LED chip junction temperature will gradually increase, the chip heat gradually increased, resulting in a substantial increase in thermal power, while the increase in optical power has gradually slowed down ${ }^{[11]}$.

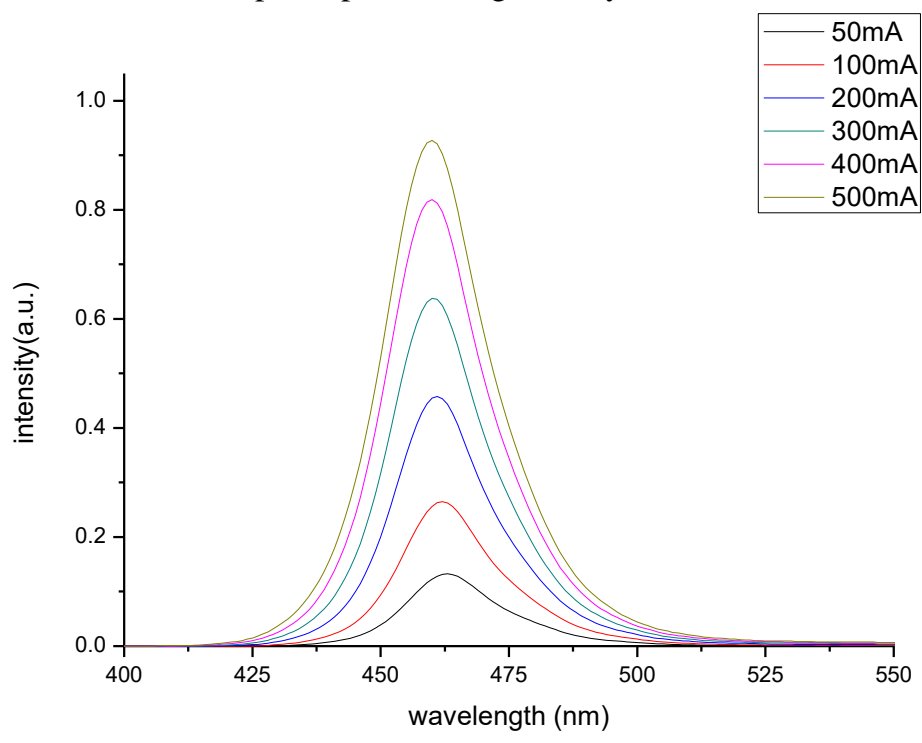

Figure 4 Excitation spectra of blue light at different currents

\subsection{Two Kinds of Test Methods Obtained by the White Spectrum}

Figure 5 for different values of the drive current, the blue LED chip directly excited yellow phosphor after the white light spectrum. Figure 6 for the different values in the drive current, the blue LED chip and yellow phosphor package, the white light spectrum. The two spectra are composed of two parts, one part of the blue LED emission spectrum; the other part of the blue light is excited by the phosphor after conversion to yellow light spectrum. In contrast to Figure 5, we can clearly find that the spectral intensities in Figure 6 are weaker than those in Figure 5. The reason is that in the packaging process, the use of packaging materials bisphenol A type epoxy resin in the case of light will be oxidized, resulting in its light transmittance decreased $^{[12]}$, by its impact, resulting in white LED after packaging Spectral energy is too small.

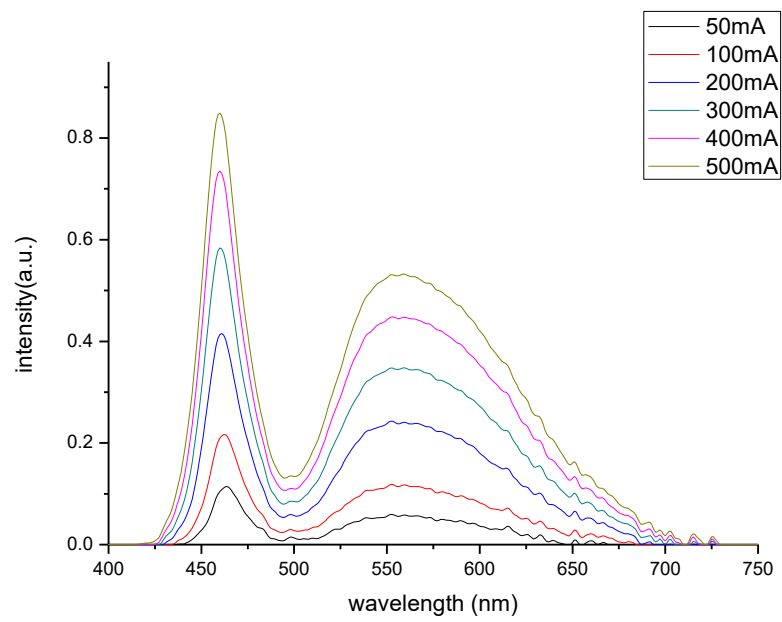

Figure 5 Spectral diagram of the white LED emitted by the direct excitation mode 


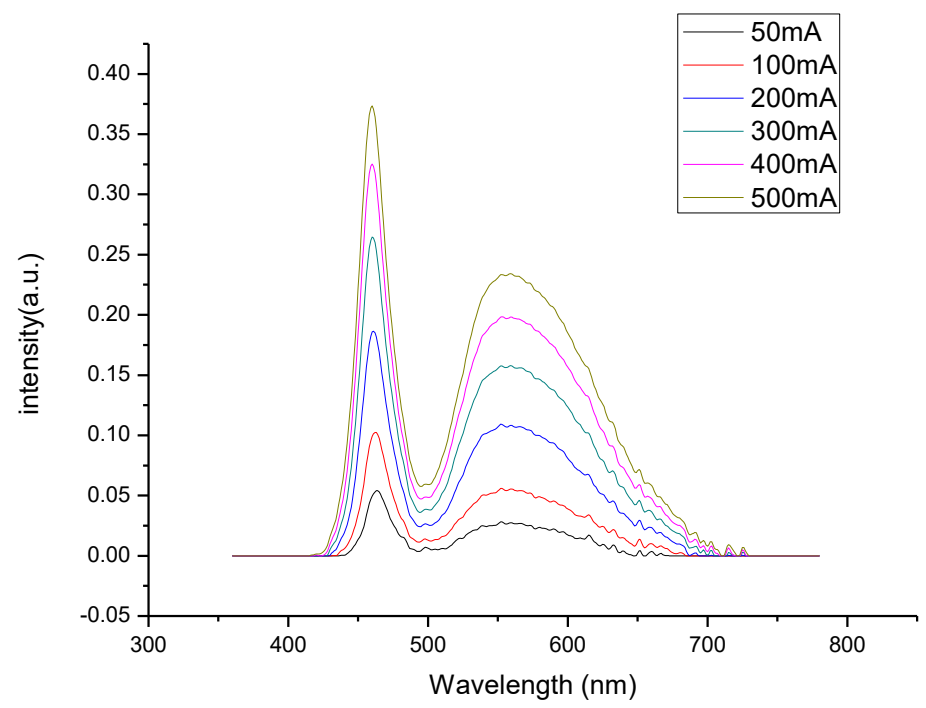

Figure 6 Measure the spectrum of the white LED emitted after encapsulation

The calculation of phosphor luminous efficiency, external quantum efficiency, energy efficiency is related to the blue excitation spectrum and the emission spectrum of the phosphor. Calculating the luminous efficacy, external quantum efficiency, and energy efficiency of the phosphor requires subtracting the remaining unconverted blue spectral portion of the excitation spectrum. Figure 7 shows the excitation spectrum of the phosphor, which is obtained by subtracting the unconverted blue spectrum from the mixed spectrum.

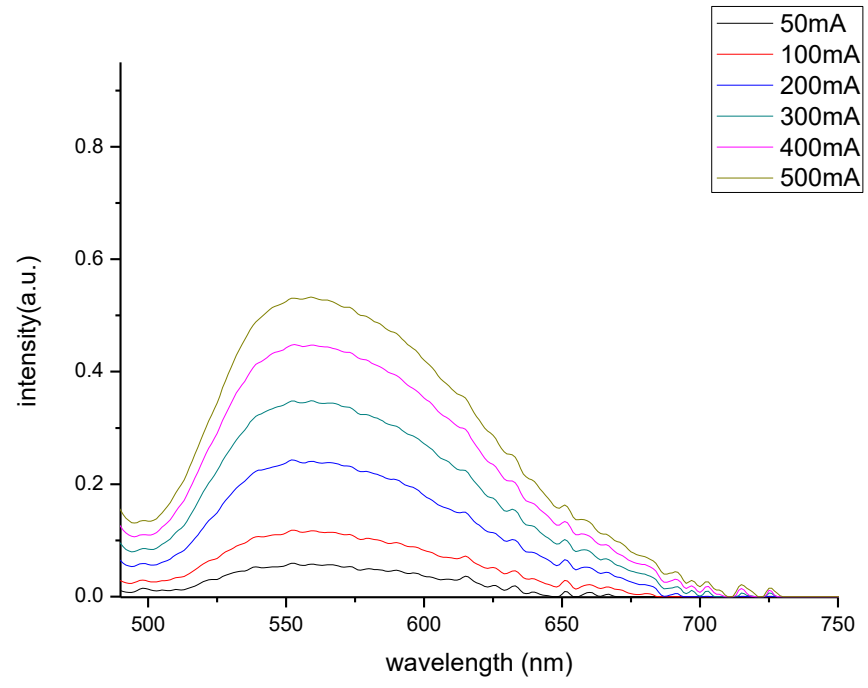

Figure 7 Excitation spectra of phosphors

\subsection{Comparison of External Quantum Efficiency, Energy Efficiency and Luminous Efficiency of Phosphor under Different Values of Current}

Figure 8 shows a comparison of external quantum efficiency and energy efficiency in two measurement modes; Figure 9 shows the comparison of luminous efficacy in two measurement modes. As can be seen from the figure, the three are generally on the increase in the trend, in the case of small current increase in the more obvious range, in the case of high current tends to be gentle, indicating that the yellow phosphor in the high current The case of light-emitting characteristics more sensitive. And compared to the two plans can be found, blue LED chip and phosphor package after the three efficiency than the direct use of direct excitation phosphor powder obtained in the three high efficiency values. This is because the epoxy resin material used under the packaging effect of light absorption, resulting in the package after the test of the luminous efficiency of the phosphor, the external quantum efficiency, energy efficiency is low. 


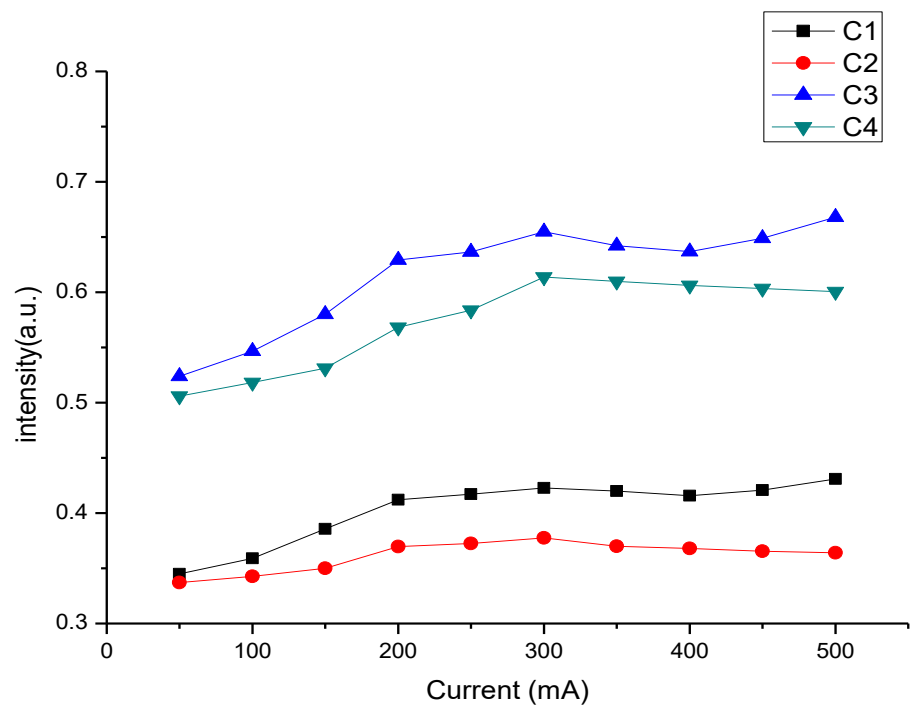

Figure 8 Comparison of energy efficiency and external quantum efficiency of the two test methods $(\mathrm{C} 1$ is the energy efficiency tested before encapsulation, $\mathrm{C} 2$ is the energy efficiency tested after encapsulation, $\mathrm{C} 3$ is the outer quantum efficiency of the pre-package test ,C4 is the external quantum efficiency of the post-encapsulated test)

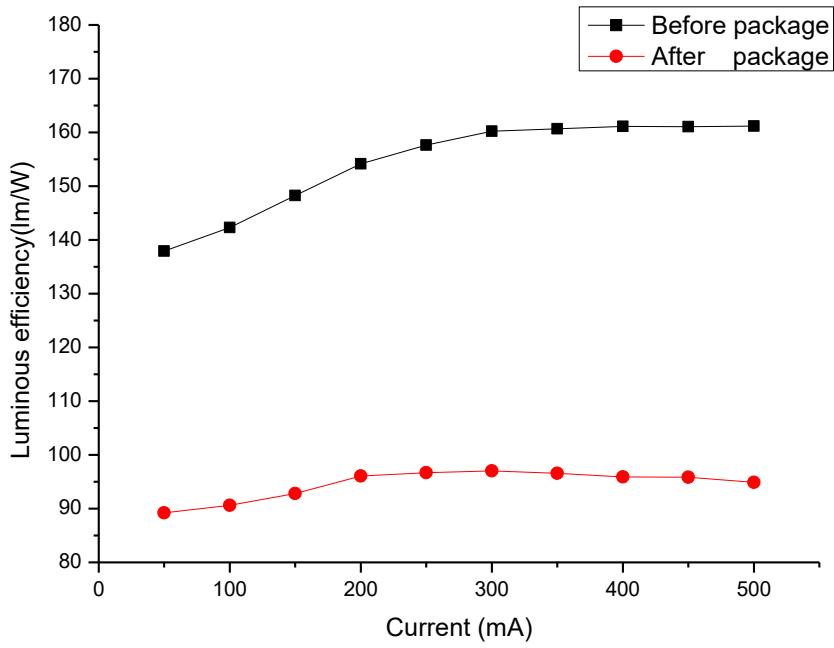

Figure 9 Comparison of luminous efficacy of two measurement methods

\section{In Conclusion}

LED as a new, energy saving and environmental protection of the "green light", with many advantages, its application prospects are further expanded, and the luminous characteristics of the phosphor is an important factor affecting the performance of LED lights. At present, there are many test systems and test methods for phosphors. Aiming at the shortcomings of traditional LED chip and phosphor packaging technology, this paper uses LED chip to direct the way of phosphor, using the integrating sphere collection device, tested 50mA, $100 \mathrm{~mA}, 200 \mathrm{~mA}, 300 \mathrm{~mA}, 400 \mathrm{~mA}, 500 \mathrm{~mA}$ and other different current Blue and white light spectrum, and with the traditional LED chip and phosphor packaging way to compare, when the LED chip with direct exposure to the phosphor, the luminous efficiency, external quantum efficiency and energy efficiency is higher, the measured luminescence Better performance. But to be more accurate test of the optical properties of phosphor, still need to further improve and improve the test method, such as the implementation of LED light source temperature control, the LED light source has been working at a specific temperature; It is possible to change the propagation path of the light source in the integrating sphere to change the propagation path of the light source.To test the luminous efficiency, external quantum efficiency and energy efficiency of the phosphor under different excitation distances, it can more accurately characterize the optical performance of the phosphor and promote the LED industry The development of LED more widely used. 


\section{References}

[1]. Chen Yuanliang, Chen Yu. LED Manufacturing Technology and Application (the second edition). Publishing House of Electronic Industry. 2009.10.

[2]. R.D.Dupuis, M.R.Krames. History,development, and applications of high-brightness visible light-emitting diodes. IEEE Journal of Lightwave Technology, 2008, 26:1154-1171.

[3]. Xiao H, Yi-Jun L U, Gao Y L, et al. Testing System of Phosphor Excited by Blue LED Based on Integral Sphere[J]. Electro-Optic Technology Application, 2013.

[4]. Chen G L, Xue H, Zhang J H, et al. White LED Phosphor Performance Tested by Spectro320e Spectrometer[J]. Advanced Display, 2012(4):29-33.

[5]. Wang Y, Mou T S, Li L. Characterization and measurement of white LED phosphors[J]. Photoelectric Engineering, 2010, 37(9):127-132.. 2006.10.03.

[6]. WANG Yi, MOU Tong-sheng, LI Li. Characterization and measurement of white LED phosphors[J]. Opto-electronic Engineering, 2010, 37 (9):127-132.

[7]. Fu W, Qian K, Luo Y, et al. Accurate determination of external photoluminescence quantum efficiency of phosphor for white LED by using inner standard scattering board[J]. Bandaoti Guangdian/semiconductor Optoelectronics, 2011, 32(5):640-645.

[8]. LIU Zong-yuan , LIU Sheng , WANG Kai, et al. Measurement and numerical studies of optical properties of YAG: Cephosphor for white light-emitting diode packaging[J]. Applied Optics, 2010, 49 (2): 247-257.

[9]. Guo-Qing W U, Guo W L, Zhu Y X, et al. Effect of driving current on the phosphor conversion efficiency of high-power white LED[J]. Journal of Optoelectronics Laser, 2012, 23(10):1869-1875.

[10]. Liu Sheng, Liu Zongyuan, Chen Mingxiang, Luo Xiaobing, Wang Kai, Gan Zhiyin. Package Structure and Packaging Method of High Power White Light Emitting Diode. Patent Application,Number 200710044964.6.

[11]. Li Liang. Influence of Phosphor Concentration and Current Intensity on Phosphor-Based White LED Characteristics[J]. Laser \& Optoelectronics Progress, 2012, 49(3):152-157.

[12]. Xu-Feng L I. Influence of Epoxy Resin upon LED Performance[J]. Chinese Journal of Luminescence, 2002. 